\title{
Correspondence between Experiment and Theory of Bulk Electrocrystallisation at Solid Electrodes in Aqueous Electrolyte
}

\section{Andersen, Jens Enevold Thaulov}

Published in:

E C S Transactions

Link to article, DOI:

$10.1149 / 1.3169316$

Publication date:

2009

Document Version

Publisher's PDF, also known as Version of record

Link back to DTU Orbit

Citation (APA):

Andersen, J. E. T. (2009). Correspondence between Experiment and Theory of Bulk Electrocrystallisation at Solid Electrodes in Aqueous Electrolyte. E C S Transactions, 16(46), 25-35. https://doi.org/10.1149/1.3169316

\section{General rights}

Copyright and moral rights for the publications made accessible in the public portal are retained by the authors and/or other copyright owners and it is a condition of accessing publications that users recognise and abide by the legal requirements associated with these rights.

- Users may download and print one copy of any publication from the public portal for the purpose of private study or research.

- You may not further distribute the material or use it for any profit-making activity or commercial gain

- You may freely distribute the URL identifying the publication in the public portal 


\title{
Correspondence between Experiment and Theory of Bulk Electrocrystallisation at Solid Electrodes in Aqueous Electrolyte
}

\author{
Jens E.T. Andersen \\ Department of Chemistry, Technical University of Denmark \\ Kemitorvet Building 207, DK-2800 Kgs. Lyngby, Denmark
}

\begin{abstract}
A model of electrodeposition and electrodissolution at electrode surfaces in aqueous solution is presented. The description is based on the assumption that redox reaction of water is the more important process controlling the electrode kinetics. Chronoamperometric measurements and experiments of cyclic voltammetry indicate that the current fundamentally is proportional to inverse time. It was proposed that redox-active species different from water never touch the surface but they predominantly interact with surface-active hydrogen or oxygen formed at the surface by redox processes of water. An excellent correspondence was found between the number of ideal-gas molecules in a monolayer at the electrode surface and the charge required, as to dissolve one monolayer of electrodeposited metal. The linear relation between standard enthalphy of metal-oxide formation and standardreduction potential shows that metal oxide formation at the electrode corresponds to oxidation of a metal in an atmosphere of oxygen.
\end{abstract}

\section{Introduction}

Polarography of reversible, irreversible and kinetic systems in experiments of cyclic voltammetry $(\mathrm{CV})$ require mathematics of a considerable degree of complexity, as reviewed by Nicholson and Shain (1). In experiments of $\mathrm{CV}$, chronoamperometry and polarography, redox processes are identified by specific peak positions in current density (2-9). Well-defined peaks in current density are obtained in systems where metallic ions are reduced at metallic surfaces more noble than the metals of the electrolyte. The reduction is particularly effective at surfaces where side reactions such as hydrogen evolution are suppressed, which occur at mercury surfaces where hydrogen evolution is kinetically hindered. Distinct peaks of redox processes are also obtained in systems of e.g. underpotential reduction, sulphide reduction at gold surfaces, hydrogen evolution at platinum surfaces and oxygen evolution at gold surfaces (7-8). At glassy carbon surfaces or modified carbon surfaces, redox processes are represented by wide peaks and broader features in the current density (9), however. Redox processes are studied within the range of potentials where the diffusion controls the rate of reaction, which corresponds to the range of potentials where redox processes of water are absent. Thus, the diffusion range is found within a range of potentials where redox processes may be studied undisturbed without the presence of water, and the peak potential represents the potential at which the process proceeds. In experiments of cyclic voltammetry, the current density peaks only in one direction of potential sweep whereas the peak is absent at the same potential when the direction of potential sweep is reversed; This discrepancy is explained by the diffusion properties of molecules adjacent to the surface of the electrode, that is, if the 
potential sweep was fast, redox-active species cannot diffuse to the electrode at a time that corresponds to the width of the diffusion layer divided by the rate of diffusion.

In the present description is modeled a situation where redox processes of water take an active part also in the redox reactions that proceeds within the diffusion range of potentials. In order to account for the basis of the model, the following assumptions are introduced:

1. Redox processes of water proceeds at all potentials in aqueous electrolytes

2. Oxygen gas or hydrogen gas evolved at the surface of the electrode diminishes the effective surface area of the electrode because the gases are entangled, adsorbed or trapped at the surface

3. Oxygen or hydrogen adsorbed to the surface at the site of water oxidation or water reduction, respectively, are the species that react chemically with redox-active species of the solution

According to these three basic assumptions, the redox-active species of the electrolyte are prevented from reaching the surface of the electrode when gaseous oxygen or gaseous hydrogen covers the surface in the form of bubbles. Once the gaseous molecules build up at the electrode, it may proceed not only at the top surface of the electrode but also within the lattice or layers of the electrode accessible to diffusion of water molecules. Accordingly, diffusion of water becomes the controlling factor in the determination of diffusion rate. In addition, since oxygen and hydrogen are relatively non-polar molecules in aqueous electrolyte they may diffuse into the surface depth of the electrode, particularly in cases where the surface also exhibits non-polar properties such as the bulk material of the glassy-carbon electrode. According to assumption \#2 (above), the bubbles of oxygen gas or bubbles of hydrogen gas may be trapped at the surface by the mere action of the gravitational-pressure force of the electrolyte, thus adding to the hydrophobic forces of attraction. The gas bubbles may be removed continuously by either rotating the electrode or by stirring the solution, as commonly introduced in electrochemical experiments. In the present investigation, however, the basic mechanisms are studied by evaluating experiments of bulk electrodeposition using stationary electrodes in stationary solutions.

\section{Experimental}

All the electrolytes were prepared by dissolution of copper sulphate, $\left(\mathrm{CuSO}_{4} \cdot 10 \mathrm{H}_{2} \mathrm{O}\right.$, Merck, p.a. CAS 7664-93-9), sulphuric acid ( $\mathrm{H}_{2} \mathrm{SO}_{4}$, Merck, p.a. CAS 7758-98-7) in distilled water. The measurements were performed at room temperature by a potentiostat/galvanostat (Gamry G300) using a three-electrode setup comprising a calomel reference electrode, a platinum counter electrode and a glassy-carbon working electrode of $3 \mathrm{~mm}$ diameter.

\section{Results and discussion}

With the precondition in mind that only a single redox reaction participates in the process, at a given potential step, the parameters of species diffusion may be determined experimentally by chronoamperometry $(3,6)$. Thus, it complicates the conventional description, if more than one reaction takes part in the process. By following the assumptions above, the basic electrochemical reactions in aqueous solution are water 
oxidation and proton reduction at anodic potentials and cathodic potentials, respectively; in acidic solution, these reactions comprise:

$$
\begin{aligned}
& 4 H^{+}(a q)+O_{2}(g)+4 e^{-} \rightleftharpoons 2 H_{2} O(l) \quad E^{0}=1.229 V \\
& 2 H_{3} O^{+}(a q)+2 e^{-} \rightleftharpoons H_{2}(g)+2 H_{2} O(l) \quad E^{0}=0 V
\end{aligned}
$$

Since the electrode is covered by oxygen or by hydrogen, the metallic ions interact with surface-active species. Reduction of the metallic ions, e.g. zinc ions, thus proceeds by chemical reaction with hydrogen at the surface:

$$
\mathrm{Zn}^{2+}(a q)+H_{2}(\text { adsorbed }) \rightleftharpoons Z n(s)+2 H^{+}(a q)
$$

The pure, metallic, finely divided and highly active metal, e.g. zinc, thus electrodeposited is labile to oxidation by oxygen in the anodic range:

$$
2 \mathrm{Zn}(s)+\mathrm{O}_{2}(\text { adsorbed }) \rightleftharpoons 2 \mathrm{ZnO}(s)
$$

According to eqs. [1] and [4], the high density of oxygen at the surface is also associated with a high density of protons, which immediately dissolve the metal oxide, e.g. zinc oxide, because acid-base reactions are fast, and the metallic ions leave the surface at the rate of diffusion, which is comparatively slow:

$$
2 \mathrm{ZnO}(s)+4 H^{+}(a q) \rightleftharpoons 2 \mathrm{Zn}^{2+}(a q)+2 \mathrm{H}_{2} \mathrm{O}(l)
$$

The net release of oxygen [1] in the anodic range may proceed in parallel with metal oxidation [4] both at the surface covered by metal and at the undeposited areas of the electrode, which shows that the current efficiency is always less than $100 \%$. That is, the charge consumed during the electrodissolution would be correspondingly larger than the molar amount of metal at the surface. The explanation for the maximum in current density of electrodissolution at certain potentials is thus related to the density of oxygen at the electrode surface; In order to become dissolved according to eqs. [4] and [5], electropositive metals need lower densities of surface oxygen whereas more noble metals require higher densities. Similarly, a low density of surface hydrogen effectively reduce species of more noble metals, and higher surface hydrogen densities are required for the reduction of ions of electropositive metals, which provides a peak in current density at different potentials.

In experiments of chronoamperometry, the metal electrodissolution at anodic potentials is identified by an increased current density vs time, as compared to the corresponding trace of the background current of the solvent. In figs. $1 \mathrm{a}$ and $1 \mathrm{~b}$ is shown the chronoamperometric measurements with a cathodic step of $-0.15 \mathrm{~V}$ in $15 \mathrm{~s}$ (Fig. 1a) followed by an anodic step of $0.15 \mathrm{~V}$ in $15 \mathrm{~s}$ (Fig. 1b) where the background signal (B) of $0.05 \mathrm{M}$-sulphuric acid was subtracted from all measurements. 

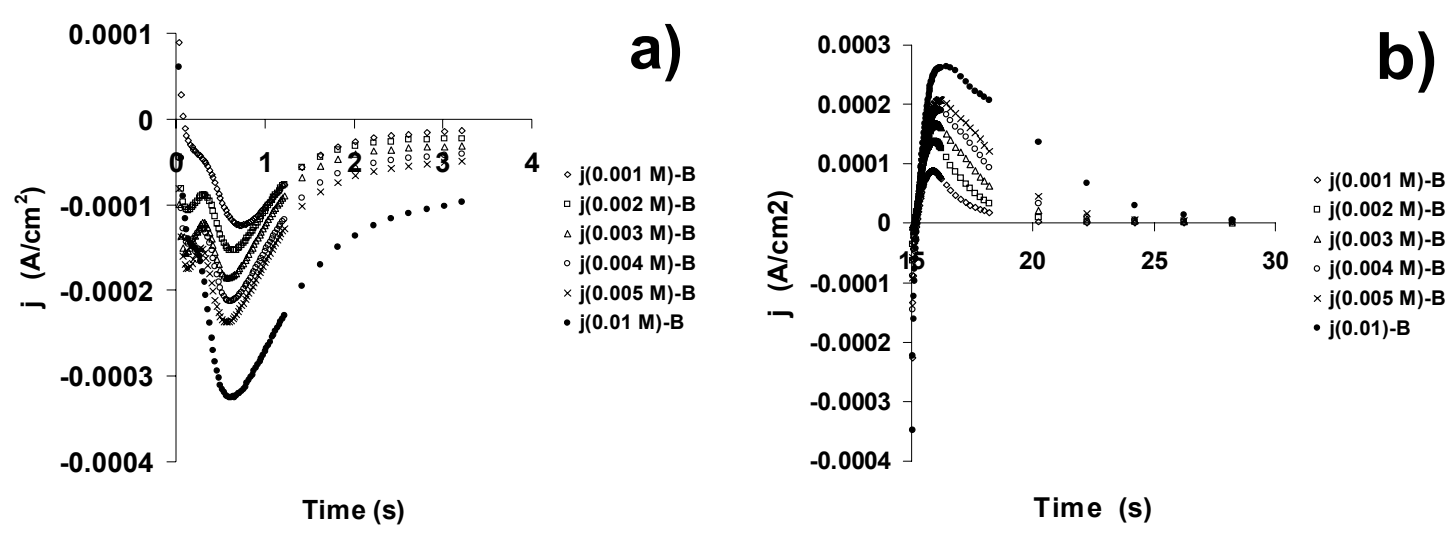

Figure 1. Chronoamperometric experiments of copper electrodepostion (a) and electrodissolution (b) on glassy carbon in an electrolyte of copper sulphate and $0.05 \mathrm{M}$ sulphuric acid at potential step values of $0.15 \mathrm{~V}$. Background (B) of sulphuric acid subtracted. The traces of current densities were recorded at concentrations of copper(II) ranging from $0.001-0.01 \mathrm{M}$, as indicated in the legends.

In both cases, the peak in current density related to copper occurs after approx. 1 second, which corresponds to a nucleation rate of approx. $1 \mathrm{~s}^{-1}$ according to the theory of Heerman and Tarallo (5). In the present context, however, the origin of the changes in current density is related to the surface being covered in molecular gas that prevents further redox reactions, that is, the area of the surface is effectively reduced, owing to the parallel production of gases that eventually cover the surface. The peak in current density of copper redox reactions may qualitatively be explained as the time where gas evolution exceeds the copper redox process, owing to a loss of surface area.

In order to estimate the amount of charge $(q)$ required for the evolution of gas at pressure $(p)$ and temperature $(T)$, the charge of the electronic circuit must correspond to the number of molecules $(n)$ of the ideal-gas equation ( $p V=n R T$, where $V$ is volume, $R$ is the gas constant and $F$ is Faraday's constant):

$$
q=I \cdot t=n \cdot F=\frac{p V}{R T} \cdot F
$$

Thus, the current becomes proportional to the inverse time:

$$
I=\frac{p A F}{R T} \cdot \frac{d}{t}
$$

where the constant surface area $(A)$ and the thickness $(d)$ of the gas slab were introduced. The surface area of the electrode thus enters the constant $k_{e}$ that is given by:

$$
k_{e}=\frac{p A F}{R T}
$$

Under standard conditions, an electrode of surface area $1 \mathrm{~cm}^{2}$ provides a value of $k_{e}=395$ $\mathrm{C} / \mathrm{m}$. The time required for the electrode to polarize the molecules thus building a Helmholtz layer is fast, as compared to the time of gas build-up at the electrode but it 
should be subtracted from the time value entering eq. 7. At a fixed potential, the potential is maintained by equilibrium between the two processes of eqs. 1 and 2, and the current may be estimated by considering the ratio between cathodic currents $\left(I_{c}\right)$ and anodic currents $\left(I_{a}\right)$ that define the surface area:

$$
\frac{I_{c}}{I_{a}}=\frac{d_{c}}{d_{a}} \cdot \frac{t-t_{a}}{t-t_{c}} \Rightarrow I_{c}=I_{a} \cdot \frac{d_{c}}{d_{a}} \cdot \frac{t-t_{a}}{t-t_{c}}
$$

where $d_{c}$ and $d_{a}$ are the thicknesses of the gas slabs in the cathodic range and in the anodic range, respectively. The time constants $t_{c}$ and $t_{a}$ denote the time for establishing the Helmholtz layer in the cathodic range and in the anodic range, respectively. By stepping the voltage as in cyclic voltammetry, the time values of eq. 9 may be substituted by potentials, $t=E \cdot d E / d t$, which transform eq. 9 into:

$$
I_{c}=I_{a} \cdot \frac{d_{c}}{d_{a}} \cdot \frac{E-E_{a}}{E-E_{c}}
$$

The maximum current is obtained at the potential $\left(E_{\max }\right)$ where oxygen gas leaves the surface in the form of bubbles or by dissolution in water. This potential corresponds to the case where the surface area is cleared from adsorbed gas and replenished by surfaceactive gas at a high rate. By introducing the potential of zero charge $\left(E_{P Z C}\right)$ where the sum of anodic current and cathodic current equals zero, the corresponding currents may be expressed as:

$$
\begin{aligned}
& I_{\text {anode }}=I_{0} \cdot \frac{d_{\text {anode }}}{d_{0}} \cdot \frac{E_{P Z C}-E}{E-E_{\max }} \\
& I_{\text {cathode }}=I_{0} \cdot \frac{d_{\text {cathode }}}{d_{0}} \cdot \frac{E}{E-E_{P Z C}+E_{\max }}
\end{aligned}
$$

Thus, the cathodic and anodic current may be estimated within the full range of potentials, as shown in fig. 2. The current expressed by the two equations 11 and 12 may thus be applied to determination of the ratio of characteristic thicknesses of gas sheaths of oxygen and hydrogen. The thickness denoted as $d_{0}$ corresponds to the current obtained in a tentatively pure ohmic response where gasses do not limit the current. These equations [11-12] correspond to the Butler-Volmer equation that accounts for the anticipated onset of hydrogen and oxygen evolution (10). In the chronoamperometric experiments of Fig. 1, the constant of eq. 8 was estimated as $3.7 \cdot 10^{-4} \mathrm{C} / \mathrm{m}$, which provides an average thickness of the gas sheath equal to 13 micron. This is a value slightly higher than the common extension of the diffusion layer $(2,3)$ but it should be noted that both hydrogen and oxygen may also be entangled with the top surface layer thus trapping gaseous species at the interface sites where water redox reactions occur. 


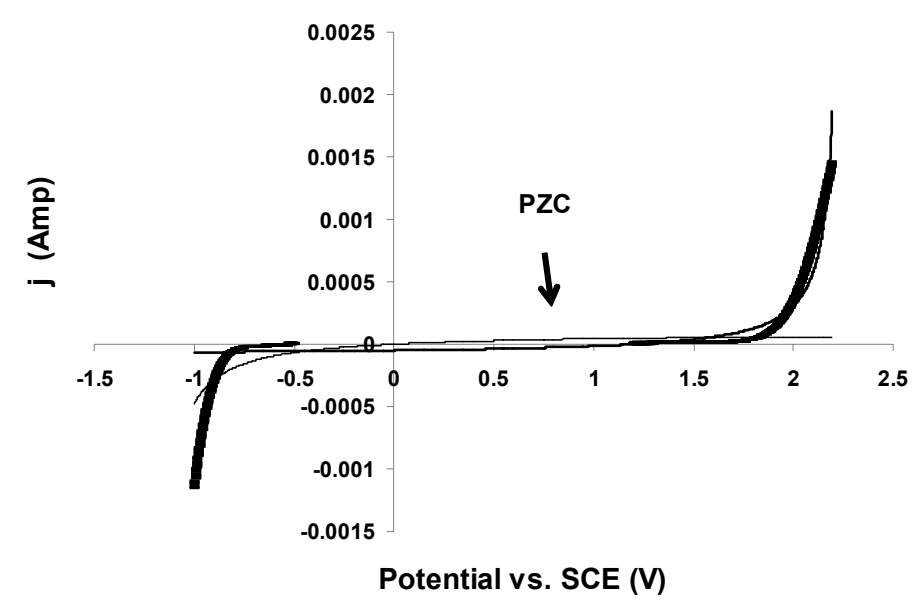

Figure 2. The background-current density of $0.05 \mathrm{M}$ sulphuric acid measured by $\mathrm{CV}$ (Thick line). Only values free of redox processes were applied, as to estimate the anodic current (Thin line, $1.2 \mathrm{~V}$ to $2.2 \mathrm{~V}$, eq. 11) and the cathodic current (Thin line, $-1.0 \mathrm{~V}$ to $0.5 \mathrm{~V}$, eq. 12).

The mechanisms of chronoamperometric experiments are illustrated schematically in Fig. 3 where the build-up of oxygen in the anodic range $(+)$ prevents ions of the electrolyte reaching the surface-active oxygen. Once oxygen escapes the surface either by dissolution or by evasion, the surface again becomes available to reaction between water or oxygen and the metal. The metal oxide thus formed rapidly dissolves by the action of acids [5] at the surface (Fig. 3).

Within the cathodic range, electrocrystallisation at low overpotentials provides diffusion controlled nucleation according to the models of Scharifker and Hills (3), Milchev (4) and Heerman and Tarallo (5). These model descriptions of the nucleation process anticipate that the ions interact directly with the electronic states of the surface and they do not consider the possibility of an intercepting layer of hydrogen chemically reducing the ions during the electrocrystallisation. However, the controlling mechanism of diffusion (3-5) still prevails even if a sheath of hydrogen covered the surface during the reduction of metallic ions. The present model thus adjusts the contemporary descriptions, and the main differences in current-density traces are expected to occur at low time values in chronoamperometric measurements.

The introduction of hydrogen at the surface mainly affects the values of the parameters of charge, diffusion coefficients and surface density (3-5). The evolution of hydrogen also has an effect on systems of self-assembled monolayers (SAM's) (6-7). It has been proposed that holes in the thiolate SAM on $\mathrm{Au}(111)$ observed by in situ STM (6) were associated with defects, such as edges, steps and kinks, on the gold crystalline surface. This explanation may be extended as to encompass the most likely sites of hydrogen evolution, which coincide with site of defects where hydrogen evolves simultaneously from two or more layers at a crystalline surface thus producing a burst of high-density hydrogen. With this suggestion in mind, the holes in the SAM (6) could be explained as vents for liberation of hydrogen through the thin-film SAM. It should be noted that hydrogen molecules in motion cannot be imaged by in situ STM, and they are not seen in images unless residing in an adsorbed state. These same vents for hydrogen liberation also account for electrocrystallisation that proceed by growth of dendrites 
underneath the SAM (6), where the dendrites growth occurs at the sites of high-density hydrogen. Once the dendrites grow in the direction perpendicular to the surface, the hydrogen evolves also from the surface of the dendrites thus forming characteristic 'mushroom-shaped' structures (7). Accordingly, it is predicted that the density of imperfections in the SAM structure never vanishes under electrochemical conditions, unless hydrogen follow some other pathways different from through-layer movement to the diffusion layer.

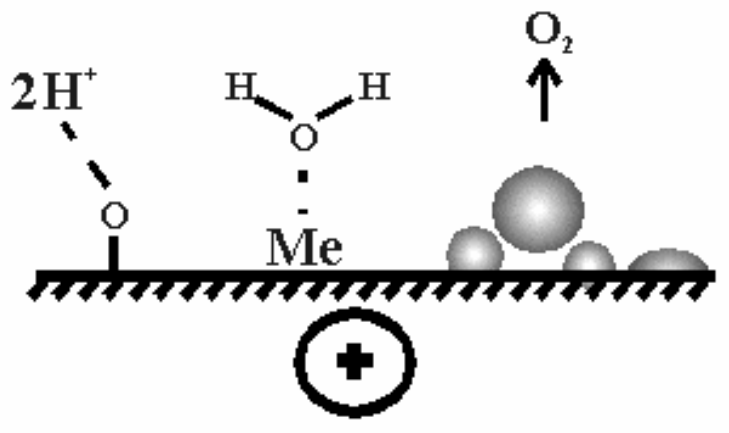

Figure 3. Schematic representation of the mechanisms responsible for oxidation of metallic ions. Metallic ions at the surface may be attacked directly by water or oxidized by surface oxygen followed by acid dissolution.

The idea of the existence of a background current that originates from the equilibrium between water reduction and water oxidation in the diffusion range may be further substantiated by subtraction of the background from the current of redox processes of sulphuric acid (Fig. 4); This procedure reveals two additional peaks of the voltammogram, which are not related to additional redox processes at the surface. The peaks in the CV's of redox processes (Fig. 4) are proposed to be related to the density of surface gas that is required to promote the process. In this view, the peaks of one specific redox reaction may occur at several different potentials, that is, the potential controls only the redox reactions of water and the redox processes of additional species of the solutions quickly removes freshly formed gas molecules that are not assembled in bubbles. 


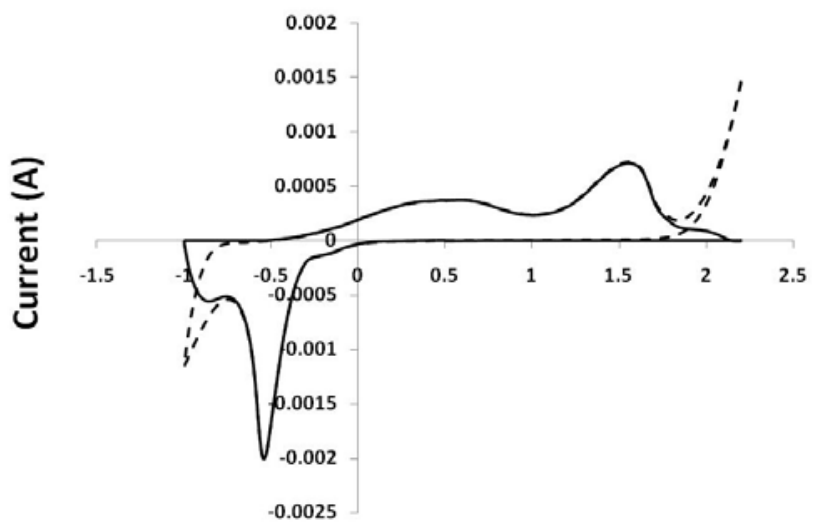

Potential vs. SCE (V)

Figure 4. Overlay plot of a CV of $0.05 \mathrm{M}$ sulphuric acid, which shows that additional peaks appear upon subtraction of the background current in potential ranges of pure hydrogen evolution and pure oxygen evolution. Original CV shown by broken line.

The additional species of the electrolyte may be copper ions in the sulphuric-acid electrolyte (Fig. 5). In order to facilitate comparison, the current densities are represented by numerical values. Addition of $1 \mathrm{mM}-10 \mathrm{mM}$ of copper yields traces of current densities that reveal copper redox reactions at several potentials both in the cathodic region and in the anodic region (Fig. 5). Further, the potential values of copper redox reactions depend on concentration, and it may be difficult to discern redox processes of both oxidation states of copper although a double-peaked structure may be associated with both the cathodic- and anodic peaks (Fig. 5). The background current related to the hydrogen and oxygen evolution proceeds when redox species of the electrolyte are prevented from reaching the surface, owing to partial-gas coverage. If the background current were subtracted (Fig. 5), the anodic charge does not correspond to the cathodic charge, owing to the non-unity of hydrogen to oxygen ratio (eqs. 11 and 12). The numerical value of the currents of the CV's after subtraction of the common background (Fig. 5) show that the presence of copper influences the current density over potential ranges both in the anodic range and in the cathodic range. A shift of potentials as a function of concentrations was also observed where the peak potentials tended to move the most at low concentrations, as seen by the more displaced peak of $0.001 \mathrm{M}$ copper(II) ions, as compared to the displacement of the other peaks of high concentrations (Fig. 5).

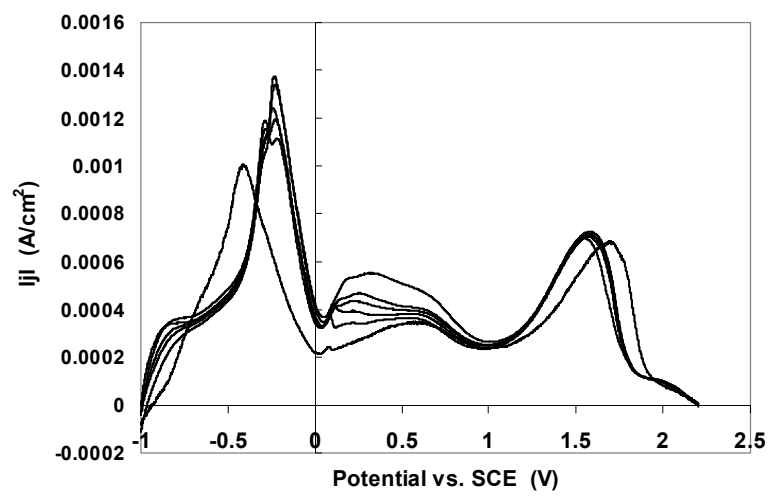


Figure 5. Numerical values of difference currents of copper electrodeposition and copper electrodissolution at glassy carbon by $\mathrm{CV}$, as obtained after subtraction of the experimental background current of oxygen evolution and of hydrogen evolution in the full range of potentials. The difference curves show that the hydrogen evolution and the oxygen evolution were affected by the presence of copper within a wide range of potentials. Sweep rate $50 \mathrm{mV} / \mathrm{s}$.

In order to correlate the amount of gas produced at the surface to the charge delivered by the potentiostat, the area-charge density may be estimated by considering the ideal gas equation and the concomitant average number of molecules in contact with the surface. It may thus be shown on the basis of eq. 6 that the area-charge density of ideal gas molecules $\rho_{q}$ may be expressed as :

$$
\rho_{q}=A \cdot F \cdot\left(\frac{4 p}{3 \sqrt{\pi N_{A}} \cdot n R T}\right)^{2 / 3}
$$

By this equation, the area-charge density of a singly charged species $n=1$ may be calculated, which yields $8 \cdot 10^{-8} \mathrm{C} / \mathrm{m}^{2}$ at an electrode-surface area of $7.1 \cdot 10^{-5} \mathrm{~m}^{2}$. Within the anodic range, oxidation of metals require at least two charges per oxygen molecule, and the surface charge required to oxidize a single layer of metal is shown in Table I for metals of valences 1-3.

Table I. The charge required to dissolve a monolayer of metal by surface oxygen at the glassy-carbon electrode of $3 \mathrm{~mm}$ diameter (eq. 13). Accidentally, this particular charge corresponds to the charge per mol in Fig. 6.

\begin{tabular}{llc}
\hline \multicolumn{1}{c}{ Reaction } & \multicolumn{1}{c}{$\begin{array}{c}\text { Charge of ideal } \\
\text { gas (x 10 }\end{array}$} & $\begin{array}{c}\text { Slope of enthalpy } \\
\text { line (C/mol) }\end{array}$ \\
\hline $4 \mathrm{M}+\mathrm{O}_{2}+4 \mathrm{H}^{+} \rightleftharpoons 4 \mathrm{M}^{+}+2 \mathrm{H}_{2} \mathrm{O}$ & 1.6 & $16300 \pm 200$ \\
$2 \mathrm{M}+\mathrm{O}_{2}+4 \mathrm{H}^{+} \rightleftharpoons 2 \mathrm{M}^{2+}+2 \mathrm{H}_{2} \mathrm{O}$ & 3.2 & $35000 \pm 200$ \\
$4 \mathrm{M}+3 \mathrm{O}_{2}+12 \mathrm{H}^{+} \rightleftharpoons 4 \mathrm{M}^{3+}+6 \mathrm{H}_{2} \mathrm{O}$ & 9.5 & $105000 \pm 3000$ \\
\hline
\end{tabular}

If the production of a metal oxide at the surface prevailed, according to eq. 4 , then the standard enthalpy of formation $\Delta H_{f}^{0}$ of the metal oxide may be related to the standard reduction potentials $\Delta E^{0}$ of the metal because it is the valence of the metal that determines the amount of oxygen consumed at the electrode. Such a correspondence was indeed present, and the depiction of standard enthalpy of formation as a function of standard reduction potentials (11) in Fig. 6 evidences that the correspondence is excellent. 


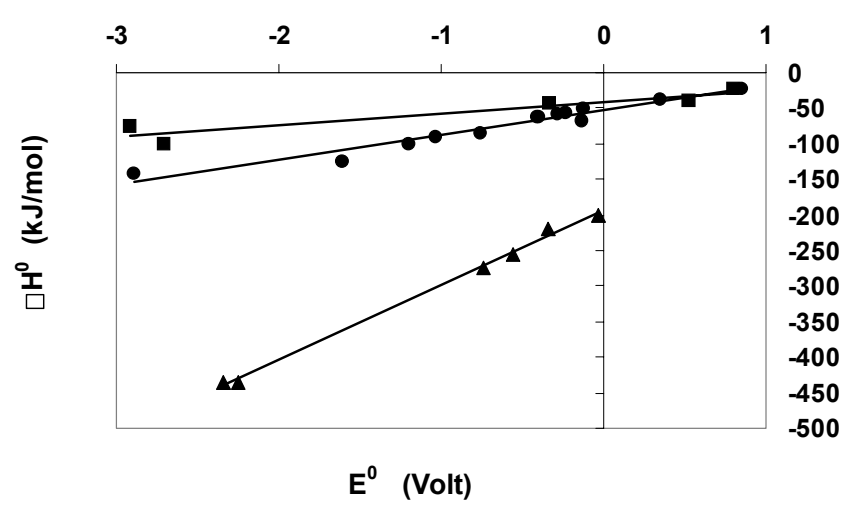

Figure 6. The enthalpy of metal oxide formation from the elements depicted as a function of reduction potentials show that they are linearly dependent. The slopes are related to the charge at the electrode surface in the anodic-potential range (see text). ( $\mathbf{a}) \mathrm{Na}_{2} \mathrm{O}, \mathrm{Cs}_{2} \mathrm{O}$, $\mathrm{Tl}_{2} \mathrm{O}, \mathrm{Hg}_{2} \mathrm{O}$ and $\mathrm{Cu}_{2} \mathrm{O}(\bullet) \mathrm{SrO}, \mathrm{VO}, \mathrm{TiO}, \mathrm{MnO}, \mathrm{FeO}, \mathrm{CoO}, \mathrm{NiO}, \mathrm{CuO}, \mathrm{ZnO}, \mathrm{CdO}, \mathrm{HgO}$, $\mathrm{SnO}$ and $\mathrm{PbO}(\boldsymbol{\Delta}) \mathrm{Ce}_{2} \mathrm{O}_{3}, \mathrm{Nd}_{2} \mathrm{O}_{3}, \mathrm{Fe}_{2} \mathrm{O}_{3}, \mathrm{Cr}_{2} \mathrm{O}_{3}, \mathrm{Ga}_{2} \mathrm{O}_{3}$ and $\mathrm{In}_{2} \mathrm{O}_{3}$.

Although the correspondence between columns 2 and 3 in Table I may seem accidental, the charge of the ideal gas at the surface is proportional to the slope of the straight lines of Fig. 6 , the constant of proportionality is $10^{11}$, which corresponds rather accurately to a monolayer of metal in the experiments used to determine the standard reduction potentials. This excellent correspondence strongly supports the idea of the formation of surface-metal oxides in the anodic-potential range. Similar considerations may be applied to hydrogen gas in the cathodic range of potentials where reduction of metal ions proceeds but metal hydrides are less well defined, as compared to metal oxides, leaving the quantification of charges less certain. However, the investigations indicate that metal ions of the electrolyte in fact never touch the surface during electrocrystallisation and the metal is oxidized by an initial formation of surface-metal oxide that is subsequently dissolved by acid-base reactions.

The standard electrode potential that is determined experimentally by extrapolation is thus interpreted as the potential where a single monolayer is oxidized under standard conditions.

\section{Conclusion}

Redox processes at the electrode were studied by electrolytes of sulphuric acid and copper ions that both constitute active redox species at the glassy-carbon surface. It was proposed that all redox reactions at electrode surfaces are controlled by gaseous-surface hydrogen and gaseous surface oxygen formed at cathodic potentials and at anodic potentials, respectively. In the present model, the metallic ions are reduced or oxidized in wide potential ranges where the density of surface-active species is sufficient to promote the reaction at a considerable rate; this explains that the current efficiency cannot reach $100 \%$. It was thus proposed that surface hydrogen and surface oxygen produced by water of the electrolyte prevails at all potentials, also within the diffusion range of potentials. When hydrogen or oxygen are produced at the surface in quantities that allow formation of bubbles, the gases start leaving the surface thus opening additional surface areas of water reaction, which is identified as the conventional potentials of gas evolution. 
It was found that the enthalphy of metal oxidation depended linearly on metal redox potentials, and the slope of the straight line corresponded to the charge of metal oxidation. An excellent correspondence was found between the charge that corresponded to the number of ideal-gas molecules of a surface monolayer at the surface and the slope of the straight line. This result evidences that electrodissolution of metals proceed via a step of metal oxide formation [4] followed by dissolution in an acid-base reaction [5]. Accordingly, the oxidation of metals at an electrode surface is directly related to the burning of metals in an oxygen atmosphere. The oxygen produced a sheath of oxygen at the electrode surface of thickness approx. 10 micron, which was comparable to the width of the diffusion layer in electrostatic models. The correspondence with conventional theories of electrode kinetics and the present description is suggested to be related to a change of focus from the electron interacting directly with the surface to the electron interacting predominantly with water molecules of the electrolyte.

\section{References}

1. $\quad$ R.S. Nicholson and I. Shain, Anal. Chem., 36, 706, 1964.

2. D. Menshkykau, I. Streeter, and R.G. Compton, J. Phys. Chem. C, 112, 14428, 2008.

3. E.J.F. Dickinson, I. Streeter, and R.G. Compton, J. Phys. Chem. B, 112, 4059, 2008.

4. B. Scharifker and G. Hills, Electrochim. Acta, 28, 879, 1983.

5. A. Milchev, J. Electroanal. Chem., 312, 267, 1991.

6. L. Heerman and A. Tarallo, J. Electroanal. Chem., 470, 70, 1999.

7. T. Baunach and D.M. Kolb, Anal. Bioanal. Chem., 373, 743, 2002.

8. S. Langerock, H. Menard, P. Rowntree and L. Heerman, Langmuir, 21, 5124, 2005.

9. A.V. Rudnev, T. Zapryanova, E.B. Molodkina, A.I. Danilov and Yu. M.

Polukarov, Russ. J. Electrochem., 44, 840, 2008.

10. J.A.V. Butler, Trans. Faraday Soc., 19, 734, 1924.

11. CRC Handbook of Chemistry and Physics, 55 ed., ed. R.C. Weast, CRC Press (1975). 